\title{
Teoria da estabilidade do equilíbrio competitivo em mercado múltiplo numa economia Arrow \& Debreu
}

Tácito Augusto Farias*

Resumo: Este artigo tem por objetivo expor o debate contemporâneo sobre as contribuições principais à teoria da estabilidade do equilíbrio competitivo em uma economia submetida a processos diversos de ajustamento: Tâtonnement $\mathrm{e}$ NonTâtonnement. Discutimos os principais resultados estabelecidos pelos autores pertinentes a literatura sobre a teoria da estabilidade do equilíbrio walrasiano. Enunciamos e discutimos alguns dos principais teoremas. E primando pela didática realizamos exemplos numéricos, quando pertinentes, alguns não estabelecidos nos artigos originais e mesmo nos textos didáticos. Realizamos comentários pertinentes aos modelos apresentados, extraídos da literatura original.

Palavras-chave: equilíbrio; estabilidade; processos.

\section{Stability theory of competitive equilibrium in multiple market on Arrow \& Debreu economy}

\begin{abstract}
This article focuses the principal contributions to competitive equilibrium stability theory on Arrow- Debreu Economy. Discuss on walrasian equilibrium stability theory: theorem principals and basic numerical examples. This article also focuses Tâtonnement e NonTâtonnemen adjustment mechanisms.
\end{abstract}

Key words: equilibrium; stability; processes.

JEL: D5 


\section{Introdução}

A literatura especializada referente à teoria da estabilidade do equilíbrio competitivo está dispersa em artigos e livros especializados (por exemplo, Arrow-Hahn 1971) o que dificulta ao leitor não versado na literatura matemática avançada - análise funcional , topologia diferencial, geometria diferencial e sistema dinâmico - alçar voo na compreensão dos textos e mesmo na questão metodológica que lá está por trás. Com o propósito de traduzir para uma linguagem mais acessível e introduzindo o leitor ao método na configuração do equilíbrio geral, acredito estar contribuindo para a divulgação e gosto pelo estudo da literatura especializada (Debreu 1959, 1976, 1984; Menezes 1993).

Nosso artigo está configurado conforme a construção de sequência de modelos: [1] primeiro,focalizamos a metodologia utilizada na elaboração do edifício de equilíbrio geral;[2] segundo,fixamos o cenário analítico construindo um modelo preliminar utilizando a metodologia adequada e neste tópico destacamos a solução analítica de exercícios - que motiva ao leitor acessar a literatura original sobre a configuração do equilíbrio geral;[3] terceiro, fixado o alicerce reconstruímos os modelos de configuração da estabilidade do equilíbrio competitivo dispersos na literatura sob a ótica de sequência encaixante das ideias e proposições principais;[4] encerramos o artigo carregando o leitor na direção dos principais programas de pesquisa em aberto, sendo que alguns correspondem à continuidade do modelo Arrow-Debreu com adições de novas ideias, à medida que outros seguem em direção oposta operacionalizando modelos de equilíbrio em concorrência imperfeita, jogos e crescimento econômico, todos tendo como foco a teoria matemática de estabilidade (Farias 1987,1999, 2007).Este artigo é uma continuação da pesquisa publicada por Farias (2007) na medida que focalizamos o período (1958-1990) que utiliza intensivamente técnicas analíticas fundamentadas nos sistemas dinâmicos para a construção da teoria da estabilidade do equilíbrio competitivo.

\section{Cenário analítico}

O tratamento analítico da teoria do equilíbrio geral sob conteúdo axiomático teve início nos anos 50, mais precisamente a partir dos estudos realizados pela equipe de pesquisadores da Comissão Cowles. A conduta de pesquisa baseava-se na construção e solução de modelos econômicos puros e aplicados no campo da teoria do equilíbrio geral, focalizando a existência, unicidade e estabilidade, concomitantes estudos sobre problemas econométricos. O conteúdo do conhecimento matemático utilizado para construir os modelos econômicos já estava consolidado: topologia geral, sistemas dinâmicos, programação matemática determinística e estocástica, análise convexa e topologia diferencial. 
Padrões da axiomática formal são estabelecidos (Eves 1990). Primeiro, o discurso contém um conjunto de termos técnicos, tais como: elementos, relações entre os elementos, operações a serem realizadas sobre os elementos, que são deliberadamente escolhidos como termos indefinidos e são denominados os termos primitivos do discurso.

Segundo, o discurso contém um conjunto de proposições sobre os termos primitivos que são livremente escolhidas como proposições indemonstráveis e são denominados os postulados (ou axiomas), P, do discurso (Eves 1990).

Terceiro, todos os outros termos técnicos do discurso são definidos por meio dos termos anteriormente introduzidos.

Quarto, todas as outras proposições do discurso são logicamente deduzidas das proposições anteriormente aceitas ou estabelecidas e são denominadas os teoremas do discurso.

Quinto, para cada teorema do discurso só uma proposição correspondente, que pode ou não ser formalmente estabelecida, existe que afirma que o teorema particular é logicamente derivado dos postulados.

Sexto, o postulado $\mathrm{P}$ implica o teorema T. E fica estabelecida a forma de realização do edifício da teoria do equilíbrio geral quando da passagem das economias na versão Walras-Hicks-Samuelson-Metzler (1945) -Lange (1944), para a economia Arrow \& Debreu nos anos 50 (Hahn 1958,1961; Negishi 1958, 1961, 1962 ; Walker 1987; Mukherji 1973, 1995.).

Estabelecida a formalização axiomática realizamos a discussão dos conceitos e proposições elementares relativos aos problemas de existência e unicidade e construímos a Economia Arrow \& Debreu com o propósito de tratar a teoria da estabilidade no período 1958 - 1960 até o instante do rompimento analítico produzido pelo trabalho de Scarf (1960), descartando o Tâtonnement sob a condição principal de substitutibilidade das mercadorias presentes no sistema econômico.

Debreu, em 1952 (apud 1995 : 50-58), escreveu um artigo que tratava da prova da existência de um equilíbrio para um sistema mais geral composto de agentes econômicos. Dois anos mais tarde, Arrow \& Debreu (1954) escreveram um artigo mais contundente que tratou da prova da existência de um equilíbrio em um modelo integrado de produção e consumo.

Na verdade, para a prova da existência de um equilíbrio geral podem ser utilizadas técnicas diversas: (1) aplicações do teorema do ponto fixo de Brouwer (Diewert 1974) e teorema do ponto fixo de Kakutani (Arrow \& Debreu 1954); (2) algoritmos combinatórios para a computação do equilíbrio geral aproximado (Scarf 1960); (3) processos diferenciais convergindo para o equilíbrio geral denominado análise global (Smale 1976a e 1976b); (4) a teoria do índice do ponto fixo de um mapeamento (Dierker 1972). 
Em equilíbrio geral, preços de equilíbrio são interpretados como aqueles preços que coordenam os planos de compra e venda de todos os agentes na economia. Walras (1874) estabeleceu apenas um tratamento superficial sobre o problema da unicidade do equilíbrio, enquanto que Wald (1951) apresentou um tratamento sistemático sobre o problema da unicidade sugerindo caminhos de prova de natureza analítica.

Com o propósito de elaborar proposições que revelam a natureza formal do problema da unicidade do equilíbrio (problema intrinsecamente ligado ao problema de estabilidade) é fundamental que se introduzam as propriedades da função excesso da demanda: Homogeneidade, Lei de Walras, Desejabilidade e Diferenciabilidade.

Duas proposições importantes são enunciadas e motivamos seu entendimento através de exercícios numéricos.

Proposição 1: $\mathrm{O}$ sistema de preço é um sistema de equilíbrio se $\mathrm{E}(\mathrm{P})=\mathrm{O}$

(Allingham 1990: 324-327) .

Exemplo 1. Seja uma economia que possua duas mercadorias e é representada pelo sistema (E1 e E2 denominamos as funções excesso de demanda para as mercadorias 1 e 2 , P1 e P2 chamamos preços das mercadorias 1 e 2),

$$
\left\{\begin{array}{l}
\mathrm{E}_{1}=-\mathrm{P}_{1}+2 \mathrm{P}_{2}-4 \\
\mathrm{E}_{2}=\mathrm{P}_{1}-\mathrm{P}_{2}+1
\end{array}\right.
$$

cujo vetor de preço solução é $\mathrm{P}=(2,3)$

Proposição 2: Se $\mathrm{P} \in \mathcal{E}$ conjunto de preços, então $\mathrm{P} \in \mathcal{E}, \forall \mathrm{t}>\mathrm{o}$, de modo que admite-se que o vetor de preço de equilíbrio $P$ seja único se $\mathrm{q} \in \mathcal{E}$ implica a $\mathrm{q}=\mathrm{t} * \mathrm{P}, \forall \mathrm{t}>\mathrm{o}$ (Allingham 1990: 324-327) .

Exemplo 2: Fixemos $\varepsilon=\{(2,3) ;(4,6)\}$. Seja $\mathrm{P}=(2,3)$ e estabeleça $\mathrm{t}=2$. Daí, faça que $\mathrm{q}=(4,6): \mathrm{q}=2 *(2,3) \therefore \mathrm{q}=\mathrm{t}^{*} \mathrm{P}$.Logo, $\mathrm{P}$ é vetor único de equilíbrio porque o seu múltiplo não é solução.

A economia é dita regular se $\mathrm{E}(\mathrm{P})$ é de posto completo $\forall \mathrm{P} \in \mathcal{E} \mathrm{O}$ número de equilíbrios em uma economia regular é finito, cuja prova é realizada pelo uso do teorema do índice de Poincaré - Hopf (Allingham 1990: 324-327; Dierker 1990: 261-268).

Do resultado acima derivamos dois corolários importantes. 
Corolário 1: O número de equilíbrios em uma economia regular é ímpar (Dierker 1990: 261-268).

Corolário 2 : Se $\mathrm{E}$ (P) é negativo para todo $\mathrm{P} \in \mathcal{E}$, então o equilíbrio é único

(Dierker 1990: 261-268).

Exemplo 3. Suponha que existe uma economia com duas mercadorias cuja

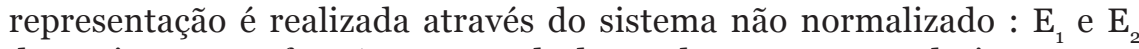
denominam-se as funções excesso de demanda para as mercadorias 1 e $2, \mathrm{P}_{1}^{2}$ e $\mathrm{P}_{2}$ são os preços das mercadorias 1 e 2, daí,

$$
\left\{\begin{array}{l}
\mathrm{E}_{1}=-\mathrm{P}_{1}+2 \mathrm{P}_{2}-3 \\
\mathrm{E}_{2}=\mathrm{P}_{1}-\mathrm{P}_{2}-1
\end{array}\right.
$$

cujo vetor de preço de equilíbrio é $\mathrm{P}=(2,3)$ quando $\mathrm{E}_{1}=0$ e $\mathrm{E}_{2}=0$. Daí, calcula-se

$$
\begin{aligned}
& |\mathrm{E}(\mathrm{P})|=\left|\begin{array}{cc}
\frac{\partial \mathrm{E}_{1}}{\partial \mathrm{P}_{1}} & \frac{\partial \mathrm{E}_{1}}{\partial \mathrm{P}_{2}} \\
\frac{\partial \mathrm{E}_{2}}{\partial \mathrm{P}_{1}} & \frac{\partial \mathrm{E}_{2}}{\partial \mathrm{P}_{2}}
\end{array}\right| \\
& |E(P)|=\left|\begin{array}{rr}
-1 & 2 \\
1 & -1
\end{array}\right| \quad \therefore \quad|E(P)|=-1 \leq 0
\end{aligned}
$$

O que implica que $\mathrm{P}=(2,3)$ é único.

Uma definição de extrema importância para o desenvolvimento da teoria da estabilidade é descrita como segue: uma economia com função excesso da demanda $\mathrm{E}$ tem a propriedade de preferência revelada se $\mathrm{P}$. $\mathrm{E}(\mathrm{q})>0$ sempre que $\mathrm{P} \in \varepsilon$ e q $\notin \varepsilon$.

Exemplo 4: Suponha uma economia que possua duas mercadorias, que é representada pelo sistema $\left(\mathrm{E}_{1}\right.$ e $\mathrm{E}_{2}$ denominados funções excesso de demanda para as mercadorias 1 e $2, \mathrm{q}_{1}$ e q ${ }_{2}$ chamados preços das mercadorias 1 e 2 ), 
daí,

$\mathrm{E}_{1}\left(\mathrm{q}_{1}, \mathrm{q}_{2}\right)=-\mathrm{q}_{1}+2 \mathrm{q}_{2}-3$

$\mathrm{E}_{2}\left(\mathrm{q}_{1}, \mathrm{q}_{2}\right)=\mathrm{q}_{1}-\mathrm{q}_{2}+1$

e faça $q=(2,5)$ um novo conjunto de preços e que

$\mathrm{E}_{1}(2,5)=5$ e $\mathrm{E}_{2}(2,5)=-2$. Daí, $\quad \mathrm{P} \cdot \mathrm{E}(\mathrm{q})=(1,2) \cdot(5,-2) \therefore$

P.E(q) $=1.5+2 .(-2) \therefore \quad$ P.E(q) $=5-4 \therefore$

$\operatorname{P.E}(q)=1>0$

Uma observação importante é que se todos os agentes são idênticos (preferências e dotações iguais) a economia tem a propriedade de preferência revelada. De fato, a razão essencial pela qual a propriedade mantém-se é que, se todos os agentes são idênticos não existe troca no equilíbrio. Este resultado é utilizado por Arrow e outros em teoremas nos artigos de 1958: Arrow-Hurwics (1958), Arrow-Macmanus (1958), Arrow-Nerlove (1958); e Arrow, Block e Hurwics (1959).

Propriedades importantes para a construção dos modelos de equilíbrio geral são estabelecidas. Na primeira, uma economia com função excesso da demanda E tem a propriedade de substituto bruto forte se $P_{i}>$ qi e $P_{j}=q_{j}$ para cada $\mathrm{i} \neq \mathrm{j}$ implicando que $\mathrm{E}_{\mathrm{j}}(\mathrm{P})>\mathrm{E}_{\mathrm{j}}(\mathrm{q}), \forall \mathrm{i} \neq \mathrm{j}$. Na segunda, uma economia com função excesso da demanda $\mathrm{E}$ tem a propriedade de substituto bruto fraco se $P_{i}>q_{i}$ e $P_{j}=q_{j}$ para cada $i \neq j$ implicando que $E_{j}(P) \geq E_{j}(q), \forall i \neq j$ (Mckenzie 1960; Hotaka 1971).

Duas proposições fundamentais são derivadas das propriedades acima. Primeiramente, a propriedade de substitutibilidade bruta implica a propriedade de preferência revelada. Em segundo lugar, a propriedade de substitutibilidade bruta implica unicidade do equilíbrio. E salienta-se que as propriedades de preferência revelada e substitutos brutos (forte e fraco) não dependem da diferenciabilidade.

Finalmente, deve-se observar que o estudo sobre a estabilidade global do equilíbrio competitivo está diretamente ligado ao método direto de Lyapunov 
que depende do uso da função Lyapunov. Diz-se que V é uma função Lyapunov se atende a três condições:

$$
\begin{array}{ll}
1 \mathrm{~V}(\mathrm{P})>0, & \mathrm{P} \neq \mathrm{P}_{\mathrm{e}} \\
3 \mathrm{~V}(\mathrm{P})<\mathrm{O}, & \mathrm{P} \neq \mathrm{P}_{\mathrm{e}} .
\end{array}
$$

E a partir da definição da função Lyapunov é possível estabelecer um resultado importante: se $\mathrm{V}>\mathrm{o}$ sempre que $\mathrm{P} \neq \mathrm{P}$, o equilíbrio é dito globalmente estável. Este resultado é amplamente utilizado na construção dos modelos de equilíbrio geral quando do tratamento na teoria da estabilidade em economias com vetor de preços globalmente estável e pressupõe que o equilíbrio é único e que este não é o caso geral da economia Arrow-Debreu.

\section{Fundamentos da teoria da estabilidade do equilíbrio competitivo - O processo de ajustamento de preços no sistema econômico: Tâtonnement}

Tâtonnement (Nikaido 1960; Arrow \& Hahn 1971; Walker 1987) é um mecanismo de ajustamento explícito que comanda a maneira pela qual preços e quantidades demandadas e ofertadas mudam no tempo em resposta a uma perturbação do equilíbrio. Sua característica principal é que a troca toma lugar somente quando um vetor de preços de equilíbrio é anunciado e a dotação inicial dos indivíduos permanece a mesma durante o período de ajustamento do sistema econômico. Este é o mecanismo de ajustamento de preços cuja origem remonta a Walras e utilizou Hicks (1939), Samuelson (1941, 1942, 1947), Lange (1944), Metzler (1945) e Arrow e outros (1956, 1958, 1959, 1960), e os economistas matemáticos japoneses Uzawa e Negishi, antes da contestação realizada no início dos anos 6o por Scarf (1960) e Gale (1960).

O estudo da teoria da estabilidade do equilíbrio competitivo baseado numa estrutura da economia Arrow \& Debreu tem seu início com os artigos de Arrow \& Hurwicks (1958), Arrow, Block e Hurwics (1959).

No primeiro artigo (1958) os autores priorizam o problema da estabilidade dinâmica de um mercado perfeitamente competitivo que possui um mecanismo de ajustamento de preços proporcional ao excesso da demanda. Formalmente,

$$
\frac{\mathrm{dP}_{\mathrm{i}}}{\mathrm{dt}}=\mathrm{K}_{\mathrm{i}} \cdot \mathrm{E}_{\mathrm{i}}\left(\mathrm{P}_{\mathrm{i}}\right)(\mathrm{Eq} \cdot 4)
$$


Elabora-se o artigo iniciando-o com a apresentação dos conceitos dinâmicos fundamentais à compreensão do problema da estabilidade dinâmica de um mercado perfeitamente competitivo: ponto de equilíbrio, equações do mecanismo de ajustamento dos preços, estabilidade local e estabilidade global.

Estabelecidos os conceitos fundamentais, Arrow \& Hurwicks (1958) descrevem os processos de ajustamento de preços que são utilizados como meio para estudar o problema da estabilidade no mercado competitivo múltiplo: processo de ajustamento instantâneo e processo de ajustamento defasado.

Exemplo 5. Suponha uma economia comandada pelo processo de ajustamento de preços

$\mathrm{dP}=\mathrm{k}$. E(P)

$\mathrm{dt}$

cujas funções de oferta $\left(S_{t}\right)$ e demanda $\left(D_{t}\right)$ são descritas abaixo,

$$
\left\{\begin{array}{l}
D_{t}=a \cdot P_{t}+b \\
S_{t}=A \cdot P_{t}+B
\end{array}\right.
$$

Substituímos as equações na definição de E(P) :

$$
E(P)=D(P)-S(P)
$$

$\mathrm{E}(\mathrm{P})=\mathrm{a} \cdot \mathrm{P}+\mathrm{b}-\mathrm{A} \cdot \mathrm{P}-\mathrm{B}$

$\mathrm{E}(\mathrm{P})=(\mathrm{a}-\mathrm{A}) \cdot \mathrm{P}+(\mathrm{b}-\mathrm{B})$

$\mathrm{dP}=\mathrm{k}[(\mathrm{a}-\mathrm{A}) \cdot \mathrm{P}+(\mathrm{b}-\mathrm{B})]$

$\mathrm{dt}$

$P=\left(P_{o}-P_{e}\right) \cdot e^{k \cdot(a-A) t}+P_{e}$

$\mathrm{P}_{\mathrm{e}}$ é dinamicamente estável pois $\mathrm{P} \rightarrow \mathrm{P}_{\mathrm{e}}$ quando $\mathrm{t} \rightarrow \infty$ se $(\mathrm{a}-\mathrm{A})<0$ com funções oferta e demanda com inclinações convencionais. 
Os resultados provenientes da elaboração do modelo com os elementos traçados nos conceitos fundamentais e mecanismo de ajustamento são colocados como segue. Primeiro, eles estudaram situações de mercado no qual não existiam troca em equilíbrio:(1) caso de indivíduos idênticos, (2) caso de uma distribuição de recurso inicial Pareto-ótimo. Segundo, elaboraram o caso para duas mercadorias. Terceiro, elaboraram um modelo que tratava do caso no qual todos os bens eram substitutos brutos em todos os preços. Realizaram uma incursão no caso em que os bens fossem considerados complementares, também.

Nas três situações consideradas foram realizados aperfeiçoamentos dos estudos anteriormente estabelecidos por Walras, Hicks, Samuelson, Lange e Metzler. O resultado fundamental derivado de todos os casos estudados: o sistema é estável sob o processo de ajustamento em um mercado perfeitamente competitivo.

A aplicação direta das definições do parágrafo anterior é caracterizada pelos seguintes teoremas:

Teorema 1: Em uma economia de troca pura, se não existe troca em equilíbrio, então o equilíbrio é localmente estável para quaisquer velocidades de ajustamento de preços.

Exemplo 6. Suponha uma economia cujas funções de oferta $\left(\mathrm{S}_{\mathrm{T}}\right)$ e demanda $\left(D_{\mathrm{T}}\right)$ sejam:

$\mathrm{D}_{\mathrm{T}}=100-5 \cdot \mathrm{P}_{\mathrm{T}}$.

$\mathrm{S}_{\mathrm{T}}=50+5 \cdot \mathrm{P}_{\mathrm{T}}$

Substituímos as equações na definição de E(P) :

$\mathrm{E}(\mathrm{P})=100-5 \mathrm{P}_{\mathrm{t}}-50-5 \mathrm{P}_{\mathrm{t}} \quad \mathrm{E}(\mathrm{P})=50-10 \mathrm{P}$

Em seguida colocamos E(P) no mecanismo de ajustamento:

$\underline{\mathrm{dE}(\mathrm{P})}=\mathrm{k} \cdot(5 \mathrm{O}-1 \mathrm{OP})$

$\mathrm{dt}$

cuja solução é descrita abaixo 
$P=\left(P_{o}-P_{e}\right) \cdot e^{k \cdot(-10 t)}+P_{e}$

e quando $\mathrm{t} \rightarrow \infty$ implica $\mathrm{P} \rightarrow \mathrm{P}_{\mathrm{e}}$, ou seja, $\mathrm{P}_{\mathrm{e}}$ é dinamicamente estável.

Este teorema é aplicado nos seguintes casos: (1) economia que possui um único consumidor; (2) economia na qual todos os consumidores possuem preferências e dotações iniciais idênticas; (3) economia na qual as dotações iniciais dos consumidores são de tal maneira que um ótimo de Pareto existe no ponto de dotação inicial.

Teorema 2: O sistema dinâmico é localmente estável e também tem estabilidade Hicksiana se todas as mercadorias são substitutos brutos. Formalmente,

$\frac{\partial \mathrm{E}_{\mathrm{i}}}{\partial \mathrm{P}_{\mathrm{i}}}=\mathrm{b}_{\mathrm{ii}}<0, \quad \forall_{\mathrm{i}}$

$\frac{\partial \mathrm{E}_{\mathrm{i}}}{\partial \mathrm{P}_{\mathrm{j}}}=\mathrm{b}_{\mathrm{ij}}>0, \quad \forall_{\mathrm{i}} \neq \mathrm{j}$

Exemplo 7: Suponha uma economia cujas funções excesso da demanda são as seguintes:

$\mathrm{E}_{2}=-4 \mathrm{P}_{2}+3 \mathrm{P}_{3}-6$

$\mathrm{E}_{3}=\mathrm{P}_{2}-\mathrm{P}_{3}-1$

1. Primeira condição: b22 < o e b33 < o

$\frac{\partial \mathrm{E}_{2}}{\partial \mathrm{P}_{2}}=-4<0$ 
$\frac{\partial \mathrm{E}_{3}}{\partial \mathrm{P}_{3}}=-1<0$

2. Segunda condição: b23 > o e b32 > o

$\frac{\partial \mathrm{E}_{2}}{\partial \mathrm{P}_{3}}=+3>0$

$\frac{\partial \mathrm{E}_{3}}{\partial \mathrm{P}_{2}}=+1>0$

e o sistema é localmente estável, cujos cálculos são descritos abaixo

$J=\left|\begin{array}{cc}-4 & +3 \\ 1 & -1\end{array}\right|=4-3 \neq 0$ : condição de existência do sistema de preços

Fazendo as equações do sistema supra descrito igual a zero :
$\mathrm{E}_{2}=\mathrm{O}$
$+4 \mathrm{P}_{2}-3 \mathrm{P}_{3}=+6$
$\mathrm{P}_{2}=3$
$\mathrm{E}_{3}=\mathrm{O}$
$\mathrm{P}_{2}-\mathrm{P}_{3}=1$
$\mathrm{P}_{3}=2$

O par $(2,3)$ é o vetor de preços de equilíbrio.

Teorema 3: Um sistema que possui um equilíbrio único e satisfaz o axioma da preferência revelada no agregado é globalmente estável. 
Exemplo 8. Considere uma economia cuja função excesso da demanda é não linear

$$
\mathrm{E}=\left(\frac{5}{\mathrm{P}}-2\right) \text { velocidade de ajustamento } \mathrm{k}=4
$$

Substituímos a equação $\mathrm{E}(\mathrm{P})$ no mecanismo de ajustamento :

$$
\frac{d p}{d t}=k \cdot\left(\frac{5}{P}-2\right)
$$

Fixando de modo conveniente a função Lyapunov:

$$
V(P)=\left(P-P_{e}\right)^{2} \quad \text { função Lyapunov. }
$$

Derivando a função Lyapunov em relação à variável tempo:

$$
\frac{d V}{d t}=2 \cdot(P-P) \cdot \frac{d P}{d t}
$$

Preparando a função derivada :

$$
\frac{\mathrm{dP}}{\mathrm{dt}}=0 \rightarrow 4 \cdot\left[\begin{array}{ll}
\frac{5}{\mathrm{P}} & -2
\end{array}\right]=0 \quad \backslash \quad \begin{aligned}
& 20 \\
& \mathrm{P}_{\mathrm{e}}
\end{aligned}-8=0
$$


$\mathrm{Pe}=2,5 \quad$ preço de equilíbrio

Substituindo o valor de P na função derivada Lyapunov:

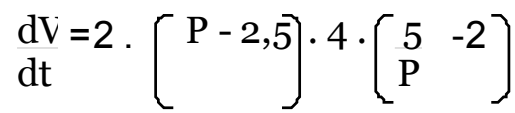

$\frac{\mathrm{dV}}{\mathrm{dt}}=8 \cdot[\mathrm{P}-2,5] \cdot\left[\frac{5}{\mathrm{P}}-2\right]<0$

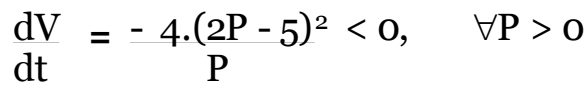

Conclui-se que $\mathrm{Pe}=2,5$ é um preço de equilíbrio dito globalmente estável.

Comentário: Assinalamos que o uso do axioma da preferência revelada é estabelecido para caracterizar o tipo de economia que foi constituída. $\mathrm{O}$ uso do método de Liapunov é compatível com uma economia com esta caracterização.

No segundo artigo, Arrow, Block e Hurwics (1959) estenderam os resultados do artigo de autoria de Arrow \& Hurwicks (1958) em várias direções. Primeiro, fornecem uma prova de estabilidade global na medida que consideram todos os bens como substitutos brutos. Este resultado mostra-se válido para processos nos quais o mecanismo de ajustamento de preço é preservador de sinal contínuo, porém não para mecanismo de ajustamento de preço no qual exista proporcionalidade em relação à função excesso da demanda. Segundo, tratam com sistemas nos quais uma das mercadorias desempenha o papel de numerário (sistema normalizado) e com sistemas nos quais todas as mercadorias são tratadas simetricamente (sistema não normalizado).

Exemplo 9: Suponha uma economia cujas funções excesso da demanda são descritas:

1. Sistema normalizado (existência de numerário). 


$$
\mathrm{P}=\left(\mathrm{P}_{1}, \mathrm{P}_{2}, \mathrm{P}_{3}\right)>\mathrm{O} \quad \begin{aligned}
& \mathrm{E}_{2}=-2 \mathrm{P}_{2}+\mathrm{P}_{3}+1 \\
& \mathrm{E}_{3}=\mathrm{P}_{2}-\mathrm{P}_{3}+1
\end{aligned}
$$

vetor de preços de equilíbrio $\mathrm{P}=(1,2,3)$.

2. Sistema não normalizado (não existência de numerário).

$$
\begin{aligned}
& \mathrm{E}_{1}=-\mathrm{P}_{1}+2 \mathrm{P}_{2}-\mathrm{P}_{3} \\
& \mathrm{P}=\left(\mathrm{P}_{1}, \mathrm{P}_{2}, \mathrm{P}_{3}\right) \geq \mathrm{O} \quad \mathrm{E}_{2}=\mathrm{P}_{1}-2 \mathrm{P}_{2}+\mathrm{P}_{3} \\
& \mathrm{E}_{3}=-\mathrm{P}_{1}+2 \mathrm{P}_{2}-\mathrm{P}_{3}
\end{aligned}
$$

vetor de preços de equilíbrio $\mathrm{P}=(2,3,4)$.

Arrow, Block e Hurwics (1959) descreveram os modelos estáticos e dinâmicos que foram utilizados como base para construírem os principais resultados.

Definiram também o preço não normalizado da k-ésima mercadoria como sendo sempre não negativo, ou seja, nenhum preço fora utilizado como numerário. Conhecido o preço estabeleceram a função excesso da demanda do i-ésimo indivíduo para a k-ésima mercadoria de modo que pudessem definir a restrição orçamentária do i-ésimo indivíduo. Assim, com o intuito de generalizar o resultado, agregaram as funções excesso da demanda individual e, deste modo, estabeleceram a função excesso da demanda agregada. Impuseram que a função excesso da demanda atendia a propriedade de ser homogênea em relação ao preço com o propósito de evitar o problema da ilusão monetária. Utilizaram esta propriedade para ambos os sistemas: normalizado e não normalizado. 
Comentários: O núcleo do artigo estabelece os elementos fundamentais referentes aos conceitos de substitutibilidade na forma incremental e na forma diferencial. A diferença entre ambas as formas é que a segunda, associada com a lei de Walras, implica na positividade do vetor preço de equilíbrio. Formalmente, estabelece a condição de substitutibilidade bruta na forma incremental, a condição de substitutibilidade bruta na forma diferencial, a condição de substitutibilidade bruta equivalente à condição de substitutibilidade bruta na forma incremental, e a condição de substitutibilidade bruta modificada.

As três primeiras condições são utilizadas na derivação dos principais resultados estabelecidos na Seção 3, enquanto que a quarta condição é utilizada na derivação do teorema 1 apresentado no artigo original.

O resultado principal sobre a estabilidade do equilíbrio competitivo sob a condição de substitutibilidade bruta é realizado. Para tornar possível a realização do resultado principal é estabelecido um roteiro:

LEMA 1: Sob as hipóteses de homogeneidade e substitutibilidade da função excesso da demanda, o vetor de preço de equilíbrio é unicamente determinado até um múltiplo escalar.

LEMA 2: Sob as hipóteses de substitutibilidade bruta, homogeneidade e lei de Walras, temos:

$$
\sum_{j} \bar{P}_{j}\left\{E_{j}(P)-\bar{E}_{j}\right\}>0
$$

para qualquer $\mathrm{P}>0, \mathrm{P} \neq \lambda \overline{\mathrm{P}}$ para qualquer $\lambda>0$.

LEMA 3: Sob as hipóteses de substitutibilidade bruta e homogeneidade, $\forall \mathrm{P}>\mathrm{O}$,

$$
\begin{aligned}
& \mathrm{P} \neq \lambda \overline{\mathrm{P}} \forall \lambda>0 \\
& \mathrm{P}_{\mathrm{k}} / \mathrm{P}_{\mathrm{k}}{ }^{\prime}=\max _{\mathrm{k}} \mathrm{P}_{\mathrm{k}} / \overline{\mathrm{P}}_{\mathrm{k}}
\end{aligned}
$$

Implica que 
$\mathrm{E}_{\mathrm{k}},(\mathrm{P})-\mathrm{E}_{\mathrm{k}}<\mathrm{O}$

$\mathrm{E}_{\mathrm{k},}(\mathrm{P})-\overline{\mathrm{E}}_{\mathrm{k},}>\mathrm{O}$

E os lemas 1, 2 e 3 são diretamente utilizados na demonstração do resultado principal:

Teorema 4: Sob as hipóteses de substitutibilidade bruta, homogeneidade e lei de Walras, o processo Tâtonnement é globalmente estável.

Exemplo 10. Imagine uma economia que possui três mercadorias, uma das quais é o numerário (P1) que é representada pelo sistema de equações a seguir:

$$
\begin{aligned}
\mathrm{E}_{2}=\frac{\mathrm{P}_{3}}{\mathrm{P}_{2}+\mathrm{P}_{3}} \\
\mathrm{E}_{3}=\frac{\mathrm{P}_{2}}{\mathrm{P}_{2}+\mathrm{P}_{3}}
\end{aligned}
$$

cujo desenvolvimento é estabelecido como segue:

1. condição de substitutibilidade bruta

$$
\begin{aligned}
& \frac{\partial \mathrm{E}_{2}}{\partial \mathrm{P}_{3}}=\frac{\mathrm{P}_{2}}{\left(\mathrm{P}_{2}+\mathrm{P}_{3}\right)^{2}}>0 \\
& \frac{\partial \mathrm{E}_{3}}{\partial \mathrm{P}_{2}}=\frac{\mathrm{P}_{3}}{\left(\mathrm{P}_{2}+\mathrm{P}_{3}\right)^{2}}>0
\end{aligned}
$$


2. Condição de homogeneidade

$$
\mathrm{E}_{2}\left(\lambda \mathrm{P}_{2}, \lambda \mathrm{P}_{3}\right)=\frac{\lambda \mathrm{P}_{3}}{\left(\lambda \mathrm{P}_{2}+\lambda \mathrm{P}_{3}\right)} \backslash \quad \mathrm{E}_{2}\left(\lambda \mathrm{P}_{2}, \lambda \mathrm{P}_{3}\right)=\mathrm{E}_{2}\left(\mathrm{P}_{2}, \mathrm{P}_{3}\right)
$$

3. Lei de Walras

$$
\begin{aligned}
& \mathrm{E}_{3}\left(\lambda \mathrm{P}_{2}, \lambda \mathrm{P}_{3}\right)=\frac{\lambda \mathrm{P}_{2}}{\left(\lambda \mathrm{P}_{2}+\lambda \mathrm{P}_{3}\right)} \backslash \mathrm{E}_{3}\left(\lambda \mathrm{P}_{2}, \lambda \mathrm{P}_{3}\right)=\mathrm{E}_{3}\left(\mathrm{P}_{2}, \mathrm{P}_{3}\right) . \\
& \sum \text { Ei.P } \mathrm{P}_{\mathrm{i}}=0 \quad \therefore \quad \mathrm{E}_{2} \mathrm{P}_{2}+\mathrm{E}_{3} \mathrm{P}_{3}=0 \frac{2 \mathrm{P}_{2} \mathrm{P}_{3}}{\mathrm{P}_{2}+\mathrm{P}_{3}}=0
\end{aligned}
$$

Comentário: Enfim, Arrow, Block e Hurwics (1959) provam a estabilidade global do mercado competitivo para um caso especial, ou seja, para o caso no qual os bens são todos considerados substitutos. Uma característica importante deste resultado é a ampla generalidade do ajustamento de preços; a taxa de mudança de preço não é necessariamente proporcional ao excesso da demanda, contudo, é assumido ser unicamente uma função preservadora de sinal do excesso da demanda.

\section{Scarf e o ataque frontal ao Tâtonnement}

A publicação dos artigos de Scarf (1960) e Gale (1960) expõem alguns resultados que contestam aqueles estabelecidos por Arrow e outros: o sistema econômico representado pelo sistema dinâmico gerado por sistemas de equações diferenciais ou diferenças são estáveis. Tanto Scarf (1960) quanto Gale (1960) apresentam exemplos numéricos convincentes em que o sistema econômico pode apresentar instabilidade quer sob a ótica local ou global.

A questão central é: o processo Tâtonnement apresenta-se geralmente estável sem quaisquer restrições tal como substitutibilidade bruta? Segundo Scarf (1960), a resposta é negativa. Imagine uma economia constituída por três indivíduos e três mercadorias e considere as funções utilidades de cada indivíduo como representadas formalmente: 


$$
\begin{aligned}
& \mathrm{U}_{1}\left(\mathrm{q}_{11}, \mathrm{q}_{12}, \mathrm{q}_{13}\right)=\min \left(\mathrm{q}_{11}, \mathrm{q}_{12}\right) \mathrm{U}_{2}\left(\mathrm{q}_{21}, \mathrm{q}_{22}, \mathrm{q}_{23}\right)=\min \left(\mathrm{q}_{22}, \mathrm{q}_{23}\right) \\
& \mathrm{U}_{3}\left(\mathrm{q}_{31}, \mathrm{q}_{32}, \mathrm{q}_{33}\right)=\min \left(\mathrm{q}_{33}, \mathrm{q}_{31}\right)
\end{aligned}
$$

com a correspondente dotação de mercadorias

$$
q_{i j}=\left\{\begin{array}{l}
1 \text { para } i=j \\
0 \text { para } i \neq j
\end{array}\right.
$$

Regra de comportamento: cada indivíduo deseja duas mercadorias que são perfeitamente complementares, ou seja, na razão de um a um. Graficamente, para quaisquer duas mercadorias:
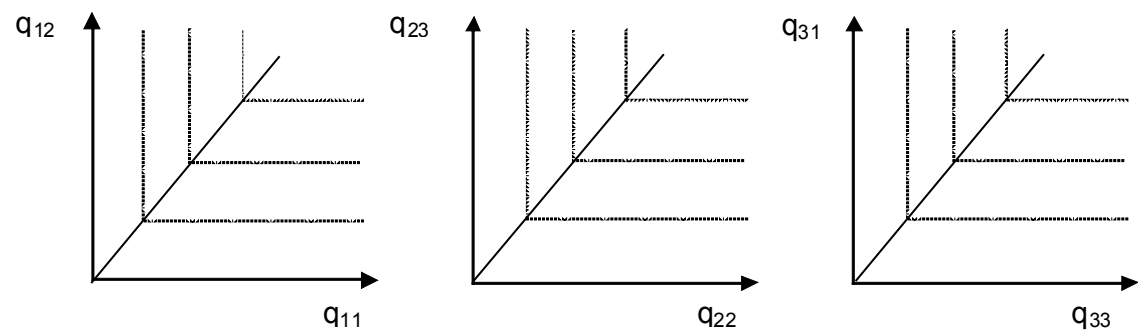

Tarefa principal : estabelecer as restrições orçamentárias dos três indivíduos e as funções excesso da demanda para cada mercadoria. Sejam $\mathrm{P}_{1}, \mathrm{P}_{2}, \mathrm{P}_{3}$ os preços das mercadorias 1, 2, 3 e sejam $\mathrm{E}_{1}, \mathrm{E}_{2}, \mathrm{E}_{3}$ as funções excesso da demanda para cada mercadoria 1, 2 e 3 , formalmente,

$\mathrm{P}_{1} \mathrm{q}_{11}+\mathrm{P}_{2} \mathrm{q}_{12}=\overline{\mathrm{P}}_{1} \mathrm{q}_{11}$ : restrição orçamentária do indivíduo 1

$\mathrm{P}_{2} \mathrm{q}_{22}+\mathrm{P}_{3} \mathrm{q}_{23}=\overline{\mathrm{P}}_{2} \mathrm{q}_{22}$ : restrição orçamentária do indivíduo 2

$\mathrm{P}_{3} \mathrm{q}_{33}+\mathrm{P}_{1} \mathrm{q}_{33}=\overline{\mathrm{P}}_{3} \mathrm{q}_{33}$ : restrição orçamentária do indivíduo 3 


$$
\begin{aligned}
& \mathrm{E}_{1}=\mathrm{q}_{1}-\overline{\mathrm{q}_{1}} \therefore \mathrm{E}_{1}=\left(\mathrm{q}_{11}+\mathrm{q}_{21}+\mathrm{q}_{31}\right)-\left(\overline{\mathrm{q}_{11}}+\overline{\mathrm{q}_{21}}+\mathrm{q}_{31}\right) \\
& \mathrm{q}_{11}=\mathrm{q}_{12} \text { e } \overline{\mathrm{q}_{11}}=1 \\
& 1 \quad \mathrm{P}_{1} \mathrm{q}_{11}+\mathrm{P}_{2} \mathrm{q}_{11}=\mathrm{P}_{1} \cdot \overline{\mathrm{q}_{11}} \\
& \mathrm{P}_{1 .}\left(\mathrm{q}_{11}-\overline{\mathrm{q}_{11}}\right)+\mathrm{P}_{2} \mathrm{q}_{12}=\mathrm{O} \therefore \mathrm{P}_{1 \cdot}\left(\mathrm{q}_{11}-1\right)+\mathrm{P}_{2} \mathrm{q}_{11}=\mathrm{O} \therefore \mathrm{P}_{1} \mathrm{q}_{11}+\mathrm{P}_{2} \mathrm{q}_{11}=\mathrm{P}_{1} \\
& \mathrm{P}_{1} \cdot\left(\mathrm{q}_{11}-1\right)+\mathrm{P}_{2} \mathrm{q}_{11}=0 \therefore \mathrm{P}_{1} \mathrm{q}_{11}+\mathrm{P}_{2} \mathrm{q}_{11}=\mathrm{P}_{1} \\
& \mathrm{q}_{11} \cdot\left(\mathrm{P}_{1}+\mathrm{P}_{2}\right)=\mathrm{P}_{1} \therefore \mathrm{q}_{11}=\frac{\mathrm{P}_{1}}{\mathrm{P}_{1}+\mathrm{P}_{2}} \\
& \mathrm{q}_{11}-\overline{\mathrm{q}_{11}}=\frac{\mathrm{P}_{1}}{\mathrm{P}_{1}+\mathrm{P}_{2}}-1 \therefore \mathrm{q}_{11}-\overline{\mathrm{q}_{11}}=\frac{\mathrm{P}_{1}-\mathrm{P}_{1}-\mathrm{P}_{2}}{\mathrm{P}_{1}+\mathrm{P}_{2}} \\
& \mathrm{q}_{11}-\mathrm{q}_{11}=-\frac{\mathrm{P}_{2}}{\mathrm{P}_{1}+\mathrm{P}_{2}} \\
& 2 \quad \mathrm{P}_{1} \mathrm{q}_{31}+\mathrm{P}_{3} \mathrm{q}_{33}=\mathrm{P}_{3} \cdot \overline{\mathrm{q}}_{33} \\
& \mathrm{P}_{1} \mathrm{q}_{31}+\mathrm{P}_{3} \mathrm{q}_{33}=\mathrm{P}_{3} \therefore \mathrm{P}_{1} \mathrm{q}_{31}+\mathrm{P}_{3} \mathrm{q}_{31}=\mathrm{P}_{3} \\
& \mathrm{q}_{31}\left(\mathrm{P}_{1}+\mathrm{P}_{3}\right)=\mathrm{P}_{3} \therefore \mathrm{q}_{31}=\frac{\mathrm{P}_{3}}{\mathrm{P}_{1}+\mathrm{P}_{3}}
\end{aligned}
$$


$3 \quad E_{1}=\left(q_{11}-\bar{q}_{11}\right)+q_{31}$

$$
\mathrm{E} 1=-\frac{\mathrm{P}_{2}}{\mathrm{P}_{1}+\mathrm{P}_{2}}+\frac{\mathrm{P}_{3}}{\mathrm{P}_{1}+\mathrm{P}_{3}}
$$

Simultaneamente,

$$
\begin{aligned}
& \mathrm{E}_{2}=-\frac{\mathrm{P}_{3}}{\mathrm{P}_{2}+\mathrm{P}_{3}}+\frac{\mathrm{P}_{1}}{\mathrm{P}_{1}+\mathrm{P}_{2}} \\
& \mathrm{E}_{3}=-\frac{\mathrm{P}_{1}}{\mathrm{P}_{3}+\mathrm{P}_{1}}+\frac{\mathrm{P}_{2}}{\mathrm{P}_{2}+\mathrm{P}_{3}}
\end{aligned}
$$

Observa-se em seguida que a condição de substitutibilidade

$$
\frac{\partial \mathrm{E}_{\mathrm{i}}}{\partial \mathrm{P}_{\mathrm{j}}}>0
$$

é violada, pois derivando-se as equações acima temos:

$$
\begin{aligned}
& \frac{\partial \mathrm{E}_{1}}{\partial \mathrm{P}_{2}}=-\frac{\left(\mathrm{P}_{1}+\mathrm{P}_{2}\right) \cdot 1-\mathrm{P}_{2}(1)}{\left(\mathrm{P}_{1}+\mathrm{P}_{2}\right)^{2}}=-\frac{\mathrm{P}_{1}+\mathrm{P}_{2}-\mathrm{P}_{2}}{\left(\mathrm{P}_{1}+\mathrm{P}_{2}\right)^{2}}=-\frac{\mathrm{P}_{1}}{\left(\mathrm{P}_{1}+\mathrm{P}_{2}\right)^{2}}<0 \\
& \frac{\partial \mathrm{E}_{2}}{\partial \mathrm{P}_{3}}=-\frac{\left(\mathrm{P}_{2}+\mathrm{P}_{3}\right) \cdot 1-\mathrm{P}_{3}(1)}{\left(\mathrm{P}_{2}+\mathrm{P}_{3}\right)^{2}}=-\frac{\mathrm{P}_{2}+\mathrm{P}_{3}-\mathrm{P}_{3}}{\left(\mathrm{P}_{2}+\mathrm{P}_{3}\right)^{2}}=-\frac{\mathrm{P}_{2}}{\left(\mathrm{P}_{2}+\mathrm{P}_{3}\right)^{2}}<0 \\
& \frac{\partial \mathrm{E}_{3}}{\partial \mathrm{P}_{1}}=-\frac{\left(\mathrm{P}_{3}+\mathrm{P}_{1}\right) \cdot 1-\mathrm{P}_{1}(1)}{\left(\mathrm{P}_{3}+\mathrm{P}_{1}\right)^{2}}=-\frac{\mathrm{P}_{3}+\mathrm{P}_{1}-\mathrm{P}_{1}}{\left(\mathrm{P}_{3}+\mathrm{P}_{1}\right)^{2}}=-\frac{\mathrm{P}_{3}}{\left(\mathrm{P}_{3}+\mathrm{P}_{1}\right)^{2}}<0
\end{aligned}
$$


que conduz ao cálculo do vetor de preço de equilíbrio

$$
\begin{aligned}
& \mathrm{E}_{1}=\mathrm{O} \Longleftrightarrow \frac{\mathrm{P}_{2}}{\mathrm{P}_{1}+\mathrm{P}_{2}}=\frac{\mathrm{P}_{3}}{\mathrm{P}_{3}+\mathrm{P}_{1}} \Longleftrightarrow \mathrm{P}_{2}=\mathrm{P}_{3}, \quad \mathrm{P}_{1}>\mathrm{O} \\
& \mathrm{E}_{2}=\mathrm{O} \Leftrightarrow \frac{\mathrm{P}_{3}}{\mathrm{P}_{2}+\mathrm{P}_{3}}=\frac{\mathrm{P}_{1}}{\mathrm{P}_{1}+\mathrm{P}_{2}} \Leftrightarrow \mathrm{P}_{3}=\mathrm{P}_{1}, \quad \mathrm{P}_{2}>0 \\
& \mathrm{E}_{3}=\mathrm{O} \Leftrightarrow \frac{\mathrm{P}_{1}}{\mathrm{P}_{3}+\mathrm{P}_{1}}=\frac{\mathrm{P}_{2}}{\mathrm{P}_{2}+\mathrm{P}_{3}} \Leftrightarrow \mathrm{P}_{2}=\mathrm{P}_{1}, \quad \mathrm{P}_{3}>\mathrm{O}
\end{aligned}
$$

$\mathrm{V}\left(\mathrm{P}_{1}, \mathrm{P}_{2}, \mathrm{P}_{3}\right)$ onde $\mathrm{P}_{1}=\mathrm{P}_{2}=\mathrm{P}_{3}$

A avaliação do comportamento do processo de ajustamento de preço é descrito a partir de

$$
\begin{aligned}
& \frac{\mathrm{dP}(\mathrm{t})}{\mathrm{dt}}=\mathrm{E}[\mathrm{P}(\mathrm{t})] \quad, \quad(\mathrm{Eq} \cdot 15) \\
& \frac{\mathrm{d}\left[\mathrm{P}_{1}{ }^{2}+\mathrm{P}_{2}{ }^{2}+\mathrm{P}_{3}{ }^{2}\right]}{\mathrm{dt}}=2 \mathrm{P}_{1} \cdot \frac{\mathrm{dP}_{1}}{\mathrm{dt}}+2 \mathrm{P} \cdot \frac{\mathrm{dP}_{2}}{\mathrm{dt}}+2 \mathrm{P}_{3} \cdot \frac{\mathrm{dP} 3}{\mathrm{dt}}
\end{aligned}
$$

Pela Lei de Walras

$$
\frac{\mathrm{d}\left(\mathrm{P}_{1} \mathrm{P}_{2} \mathrm{P}_{3}\right)}{\mathrm{dt}}=\mathrm{P}_{2} \cdot \mathrm{P}_{3} \cdot \frac{\mathrm{dP}}{\mathrm{dt}}+\mathrm{P}_{1} \cdot \mathrm{P}_{3} \cdot \frac{\mathrm{dP}}{\mathrm{dt}}+\mathrm{P}_{1} \cdot \mathrm{P}_{2} \cdot \frac{\mathrm{dP}}{\mathrm{dt}}
$$




$$
\begin{aligned}
& \frac{\mathrm{d}\left(\mathrm{P}_{1} \mathrm{P}_{2} \mathrm{P}_{3}\right)}{\mathrm{dt}}=\mathrm{P}_{2} \mathrm{P}_{3} \mathrm{E}_{1}+\mathrm{P}_{1} \mathrm{P}_{3} \mathrm{E}_{2}+\mathrm{P}_{1} \mathrm{P}_{2} \mathrm{E}_{3} \\
& \frac{\mathrm{d}\left(\mathrm{P}_{1} \mathrm{P}_{2} \mathrm{P}_{3}\right)}{\mathrm{dt}}=0
\end{aligned}
$$

Resultante das equações estabelecidas acima temos: (1) não existe efeitosubstituição, (2) a curva de indiferença não é estritamente côncava à origem, (3) a curva de indiferença tem uma quebra e portanto não é diferenciável,(4) existe somente efeito-renda.

É conhecido que Scarf (1960) impõe uma difícil e amarga derrota momentânea ao mecanismo de ajustamento de preços - Tâtonnement - quando apresenta situações em que as hipóteses sob as quais o modelo ortodoxo se assentava, substitutos brutos ou axioma fraco da preferência revelada ou diagonais dominantes falhavam à medida que o sistema econômico abstrato não convergia para o vetor de preços de equilíbrio. Como uma maneira de contornar esta situação, foram elaborados inúmeros trabalhos que contribuíram de forma decisiva para recuperar o prestígio do processo de ajustamento ortodoxo, porém, não mais utilizando-se hipóteses generalistas, e sim, estudos de casos. Destacam-se: Uzawa (1960, 1961, 1962); Arrow (1961, 1962, 1971); Quirk (1970); Habibagahi e Quirk (1973); Hotaka (1971); Gordon (1974); Smale (1976); Tarr (1978); Kamyia (1990) e Mukherji (1973, 1995). 


\section{A teoria da estabilidade no sistema econômico após o ataque frontal de Scarf}

Nesta Seção a abordagem consiste dos pontos a seguir. Primeiro, é feita a apresentação e discussão detalhada dos principais mecanismos de ajustamentos de preços Non-Tâtonnement: (1) Processo de Edgeworth caracterizado e discutido em Uzawa (1962) e Hahn (1962); (2) Hahn \& Negish (1962); (3) Green (1974). Segundo, realiza-se uma incursão na aplicação da análise global no campo da teoria do equilíbrio geral, incluindo um exercício numérico.

\subsection{Fundamentos do pro- cesso de ajustamento de preços no sistema econômico: Non-Tâtonnement}

Face aos resultados encontrados, Scarf (1960) e Gale (1960), economistas matemáticos, trataram de desenvolver um mecanismo de ajustamento explícito no qual a troca ocorre em cada valor anunciado do vetor de preço e a dotação dos indivíduos participantes do mercado muda continuadamente ao longo do processo de ajustamento do sistema, que denominaram NonTâtonnement. Dois processos de ajustamentos dos sistemas de preços na versão Non-Tâtonnement foram estabelecidos e se tornaram clássicos: Processo de Edgeworth em Uzawa (1962) e Hahn (1962), Processo de Hahn \& Negishi (1962) e Green (1974) .

O processo de ajustamento do preço Uzawa (1962) e Hahn (1962) fundamentase na hipótese de que cada indivíduo participa na troca à medida que a mesma incrementa a satisfação do indivíduo. Portanto, é admitido que a utilidade do estoque de mercadorias mantido pelos consumidores individualmente aumenta ao longo do período de tempo como um resultado das transações. Em vista da hipótese estabelecida pelo processo Uzawa (1962) e Hahn (1962), é possível traçar algum comentário pertinente ao modelo. Primeiro, a hipótese exige grande quantidade de informação do sistema econômico, facilitando a construção de exemplos nos quais somente a troca pareto-ótimo exige a participação de muitos agentes econômicos. Segundo, a hipótese de que troca raramente ocorre quando participantes, individualmente, aumentam utilidade é somente um atrativo quando agentes econômicos são considerados ingenuamente esperando por preços constantes e transações finalizadas. 
O processo de ajustamento de preço de Hahn \& Negishi (1962) está baseado na hipótese de que se existe um excesso de oferta de uma determinada mercadoria, então todos os compradores potenciais desta mercadoria podem realizar seus desejos. Ademais, se existe um excesso da demanda para uma determinada mercadoria, então todos os vendedores potenciais podem realizar seus desejos. Contudo, se os mercados são suficientemente bem organizados não existirá excesso de qualquer ordem. Um resultado importante é derivado da hipótese do modelo:

Teorema 5 - No sistema econômico, a troca ocorre somente quando o sinal do excesso da demanda para qualquer mercadoria individual é o mesmo do excesso da demanda para aquela mercadoria no agregado (Hahn \& Negishi 1962 ).

Exemplo 11. Seja uma economia, que possua duas mercadorias, representada pelas equações:

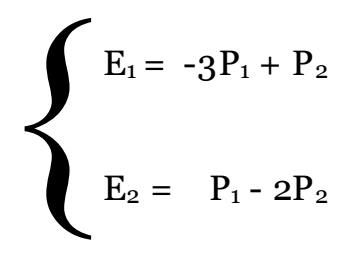

e as derivadas parciais primeiras das funções excesso da demanda individuais, 
$\frac{\partial \mathrm{E}_{1}}{\partial \mathrm{P}_{1}}=-3<0 \quad$ e $\quad \frac{\partial \mathrm{E}_{2}}{\partial \mathrm{P}_{2}}=-2<0$

E, em seguida, agregam-se as funções excesso da demanda individual e obtém-se

$\mathrm{E}\left(\mathrm{P}_{1}, \mathrm{P}_{2}\right)=\sum \mathrm{E}_{\mathrm{i}} \therefore \mathrm{E}\left(\mathrm{P}_{1}, \mathrm{P}_{2}\right)=\left(-3 \mathrm{P}_{1}+\mathrm{P}_{2}\right)+\left(\mathrm{P}_{1}-2 \mathrm{P}_{2}\right)$

$E\left(P_{1}, P_{2}\right)=-2 P_{1}-P_{2}$

Deriva-se a função excesso da demanda agregada e obtêm-se

$$
\frac{\partial \mathrm{E}}{\partial \mathrm{P}_{1}}=-2<0 \quad \frac{\partial \mathrm{E}}{\partial \mathrm{P}_{2}}=-1<0
$$

que atendem ao resultado esperado.

O professor Green (1974) descreveu o processo pelo qual a solução final era alcançada através de uma sequência de lances. Cada lance é comparado a um contralance que representa uma alocação viável que poderia ser utilizada para bloqueá-lo. Na verdade, nenhum bloqueio adicional foi possível para que o lance final fosse realizado pelos indivíduos que constituíam o sistema. Nenhuma atividade de fato acontecia enquanto o processo de recontratação ainda estivesse por estabelecer-se. Com efeito, o conjunto de todas as combinações de utilidade viabilizada pelos membros da coalizão eram fixados ao longo do curso da barganha. Este fato é derivado do processo Walrasiano Tâtonnement no qual os excessos da demanda são registrados pelos usos de tickets, e preços ajustam-se à medida que o excesso da demanda converge para zero — somente então a troca ocorre. Conclui-se que os resultados da teoria da estabilidade tradicional referente ao processo Tâtonnement estabelecem que quando $t \rightarrow \infty$ preços tornam-se arbitrariamente próximos dos preços de equilíbrio. Significa dizer que sob a ótica Tâtonnement, os indivíduos realizarão de fato qualquer troca desde que eles devam sempre esperar que os preços convirjam para o vetor de preços de equilíbrio. Assim sendo, o resultado obtido por Green é de natureza diferente daquele estabelecido pelo Tâtonnement. Ou seja, a probabilidade que o lance atual não esteja no core aproxima-se de zero quando t cresce. Portanto, a realização da troca de 
mercadorias por mercadorias, barganha, tomará lugar num período finito de tempo com certeza (probabilidade 1) o que implica o processo Tâtonnement como hipótese bem mais simples a tolerar.

\subsection{Análise global: Stephen Smale}

Professor Stephen Smale, matemático profissional, Medalha Field (1966), prêmio equivalente ao Prêmio Nobel, dá uma guinada importante em sua atividade acadêmica. Passa a atuar na aplicação da matemática pura ao desenvolvimento da teoria do equilíbrio geral. Com efeito, aponta as vantagens do enfoque da análise global aplicado à teoria do equilíbrio clássico (Stephen Smale 1976a , 1976b).

Primeiramente, as provas de existências são consideradas mais simples. $\mathrm{O}$ teorema do ponto fixo de Kakutani não é usado; o principal instrumento analítico é o cálculo das funções reais de várias variáveis reais.

Em segundo lugar, a estática comparativa é integrada ao modelo em uma maneira simples, as primeiras derivadas desempenham um papel fundamental.

Em terceiro lugar, o enfoque do cálculo está mais próximo das tradições da teoria do equilíbrio competitivo clássico.

Em quarto lugar, oportunamente, as provas de equilíbrio são construtivas. Tais provas podem ser elaboradas por um algoritmo rápido,que é o método de Newton modificado para estabelecer convergência global. Ainda as provas de existências são surpreendentemente poderosas para produzirem a generalidade da teoria axiomática Arrow \& Debreu.

Assim, estabelecem-se propriedades sobre a função excesso da demanda que são importantes na elaboração da estrutura do sistema econômico: Lei de Walras, Homogeneidade e Condições de fronteira; que geram resultados importantes.

Teorema 6 - Suponha que uma função excesso da demanda E satisfaz a Lei de Walras, homogeneidade e a condição de fronteira.

(Existência) Então existe um preço de equilíbrio.

Teorema 7 - Se uma função F: $\mathrm{U} \rightarrow \mathrm{R}^{\mathrm{n}}$, $\left(\mathrm{U}\right.$ subconjunto de $\left.\mathrm{R}^{\mathrm{K}}\right)$ é de classe suficientemente diferenciável $\left(\mathrm{C}^{\mathrm{r}}, \mathrm{R}^{\mathrm{r}}>\mathrm{K}-\mathrm{n}, \mathrm{o}\right)$ então o conjunto (teorema de Sard) de valores regulares de F tem medida completa. (Palis Jr \& Melo 1977: 3; Milnor 1965:10; Sard 1942: 883-890; Morse 1939: 62-70; Debreu 1995: 179-185). 
Teorema 8 - Se $\mathrm{y} \in \mathrm{R}^{\mathrm{m}}$ é um valor regular de uma função contínua (Função Inversa) $\quad \mathrm{F}: \mathrm{U} \rightarrow \mathrm{R}^{\mathrm{n}}$, U subconjunto de $\mathrm{R}^{\mathrm{K}}$, então ou $\mathrm{F}^{-1}(\mathrm{y})=\mathrm{o}$, ou é uma subvariedade de dimensão (K-n).

Conhecida a estrutura matemática do sistema econômico abstrato é possível então aplicar o instrumental da análise global a uma economia de troca pura. Imagine uma economia de troca na qual existe a função utilidade $\mathrm{U}$ : $\mathrm{P} \rightarrow \mathrm{R}$ e o espaço de mercadorias $\mathrm{P}=\left\{\mathrm{x} \in \mathrm{R}^{\mathrm{l}} ; \mathrm{x}>\mathrm{O}\right\}$. Tal economia é construída sob as hipóteses clássicas: Monotonicidade, Convexidade e Condição de fronteira.

De posse das hipóteses clássicas é possível gerar o resultado principal:

Teorema 9- A função da demanda F satisfaz

$1 \quad \nabla \mathrm{U}(\mathrm{F}(\mathrm{P}, \mathrm{W}))=\lambda \mathrm{P}$, para algum $\lambda>0$

$2 \quad$ P.F $(\mathrm{P}, \mathrm{W})=\mathrm{W}$

$3 \quad \mathrm{~F}(\lambda \mathrm{P}, \mathrm{W})=\mathrm{F}(\mathrm{P}, \mathrm{W}), \quad \forall \lambda>\mathrm{O}$

4 F é contínua.

Define-se o estado de uma economia como a m-upla $\left(\mathrm{x}_{1}, \mathrm{x}_{2},--, \mathrm{x}_{\mathrm{m}}\right)$ onde $\mathrm{x}_{\mathrm{i}} \in \mathrm{P}$ e $\mathrm{P} \subset \mathrm{R}$, é possível enunciar duas ordens de condições de funcionamento do sistema econômico. Quais sejam,

1 Condição de viabilidade: $\sum \mathrm{x}_{\mathrm{i}}=\sum \mathrm{e}_{\mathrm{i}} \rightarrow$ dotação inicial

2 Condição de satisfação de um estado: $\forall \mathrm{i}, \mathrm{x}_{\mathrm{i}}$ maximiza $\mathrm{U}_{\mathrm{i}}$ no conjunto orçamentário $B=\left\{y \in P: P . y=P . e_{i}\right\}$.

Para a economia de troca sob as condições supraestabelecidas gera-se um resultado importante:

Teorema 10 -Existe um preço de equilíbrio de toda economia de troca pura. 
Exemplo 12. Imagine uma economia representada pelas funções excesso da demanda:

$\left\{\begin{array}{l}\mathrm{E}_{1}=\mathrm{P}_{1}+\mathrm{P}_{2}-6 \\ \mathrm{E}_{2}=\mathrm{P}_{1}-\mathrm{P}_{2}-2\end{array}\right.$

cujos cálculos são estabelecidos como segue:

$$
|\mathrm{E}(\mathrm{P})|=\left|\begin{array}{ll}
\frac{\partial \mathrm{E}_{1}}{\partial \mathrm{P}_{1}} & \frac{\partial \mathrm{E}_{1}}{\partial \mathrm{P}_{2}} \\
\frac{\partial \mathrm{E}_{2}}{\partial \mathrm{P}_{1}} & \frac{\partial \mathrm{E}_{2}}{\partial \mathrm{P}_{2}}
\end{array}\right| \quad \therefore \quad|\mathrm{E}(\mathrm{P})|=\left|\begin{array}{cc}
1 & 2 \\
1 & -1
\end{array}\right| \quad \therefore \quad|\mathrm{E}(\mathrm{P})|=-3 \neq 0
$$

onde o jacobiano da transformação diferente de zero corresponde à condição de existência do vetor de preços de equilíbrio em uma economia de troca pura.

E a prova de existência utilizando o método de análise global estende a demonstração estabelecida pelo teorema Arrow \& Debreu, ou seja, generaliza o resultado estabelecido na estrutura da economia Arrow \& Debreu.

Em relação ao problema da estabilidade, a análise global do Professor Stephen Smale traça importantes contribuições nos anos 70, sobre a teoria da estabilidade sob o equilíbrio competitivo. Os trabalhos mais importantes de Smale sobre processos dinâmicos consistem de dois artigos escritos em 1976. 
O primeiro artigo contém um modelo de Non-Tâtonnement que segue a linha de pesquisa do modelo Hahn-Negishi (1962). Contudo, existem duas diferenças entre eles: (1) o modelo discute o processo de ajustamento de natureza estocástica e portanto é descrito por uma equação diferencial estocástica; (2) a hipótese de otimização de longo prazo é evitada. Sugere hipóteses: (a) troca ocorre aos preços correntes, (b) toda troca aumenta a satisfação dos indivíduos envolvidos, (c) alguma troca ocorrerá sempre, desde que consistente com todos os indivíduos envolvidos. Resultado: em um mercado de troca pura um processo de ajustamento específico converge a um vetor de preços de equilíbrio.

O segundo artigo contém um modelo Tâtonnement, que cobre uma nova linha de pesquisa no conhecimento e está ligado aos problemas de calcular o equilíbrio e à procura por um processo de ajustamento com melhores propriedades de convergência do que o Tâtonnement clássico. Resultado:

Teorema 11 - Exceto para um conjunto de pontos de fronteira com medidas de Lebesgue zero, toda trajetória partindo da fronteira, ou mesmo de um ponto próximo da fronteira, converge em direção ao conjunto de equilíbrio.

\section{Considerações finais}

Modelos de equilíbrio geral em mercados competitivos já estabelecidos na literatura ao longo dos anos 60,70 e 80 versavam sobre: (1) equilíbrio geral com mercados completos e informação simétrica; (2) equilíbrio geral com externalidades e bens públicos; (3) equilíbrio geral considerando a escolha sob condição de risco; (4) equilíbrio geral com mercado incompleto sob expectativas racionais; (5) equilíbrio geral sob condição de informação assimétrica e destruição dos mercados; (6) estabilidade de mercado competitivo sob condição de substitutibilidade bruta fraca em mecanismo de ajustamento de preço não linear e expectativas adaptativas; (7) estabilidade em mercado competitivo sob condição de substitutibilidade bruta fazendo uso do enfoque da distância euclideana. Linhas de pesquisas desenvolvidas ao longo dos anos 90 destacaram: (1) equilíbrio geral com mercados incompletos;(2) o papel da moeda nos modelos de equilíbrio geral com informação assimétrica;(3) equilíbrio geral sob completamento de mercados com ativos financeiros;(4) equilíbrio geral sob escolha envolvendo risco; (5) equilíbrio geral sob condição de seleção adversa. Em verdade, o desenvolvimento dos modelos de equilíbrio geral na visão da análise global é a virada de mesa na metodologia sobre a pesquisa matemática aplicada ao campo da economia pura nos anos 70 e 80 e extensivo aos anos 90. 
Enfim, as estruturas econômicas desenvolvidas pelos economistas ao longo dos anos 90, e início do próximo milênio direcionaram seus trabalhos em teoria dos jogos, especificamente estabilidade do equilíbrio estratégico e jogos sinalizados de equilíbrio estável, caos; nas teorias do crescimento econômico e ciclos de negócios vários problemas concentram a atenção dos pesquisadores ao longo dos anos 90: (1) estabilidade assintótica global e (local) de sistemas de controle ótimo aplicados à teoria do crescimento econômico; (2) procura da existência de solução para sistemas dinâmicos Hamiltoníanos de crescimento econômico ótimo; (3) relacionamento entre crescimento balanceado e eficiência intertemporal na acumulação de capital; (4) descrição da estrutura e estabilidade de sistemas dinâmicos competitivos. Com efeito, fica evidenciado que a depender da estrutura de mercado que se está trabalhando e associado às hipóteses de comportamento dos agentes econômicos individuais, observam-se resultados referentes à estabilidade do equilíbrio competitivo de maneira diversa.

\section{Referências}

ALLINGHAM, M. (1990). “Unicidade de equilíbrio”.In: EATWELl,J \& MILGATE,M \&. Newman, P. (eds).The new palgrave:General Equilibrium.New York: W.W.Norton, pp.324- 327.

ARROW, K. J.; BLOCK, H. D.; HURWICZ, L. (1959). “On the Stability of the Competitive”, part. 2. Econometrica, 27: 87-109.

ARROW,K.J.;DEBREU,G.(1954)."Existence of an Equilibrium for a

Competitive Economy”.Econometrica, 22: 265-290.

ARROW, K. J.; ENTHOVEN,A. C. (1956). "A Theorem on Expectations and the Stability of Equilibrium”. Econometrica, 24: 288-293.

ARROW K.J.;HURWICZ,L.(1960).” Competitive Stability under Weak

Gross Substitutibility: The Euclidean Distance Approach”.International Economic Review: 38-49.

ARROW, K. J.; HURWICZ, L. (1962). “Competitive Stability under Weak Gross Substitutibility:Non-linear Price Adjustment and Adaptive Expectations”. International Economic Review, 3(2) 233-255.

ARROW, K. J.; HURWICS, L. (1960). "Some Remarks on the Equilibria of Economic Systems.”Econometrica, 28: 640-646. 
ARROW,K.J.;HURWICS,L.(1958).”On the Stability of the Competitive Equilibrium”, part1.Econometrica, 26: 522-552.

ARROW,K.J.;HAHN F.H.(1971).General Competitive Analysis. San Francisco: Holden-Day.

ARROW,K.J.;MACMANUS,M.(1958). “A Note on DynamicStability”. Econometrica, 26: 297-305.

ARROW,K.J.;NERLOVE,M.(1958). “A Note on Expectation and Stability”. Econometrica, 26: 297-305.

DEBREU,G.(1959).Theory of Value:An Axiomatic Analysis of Economic Equilibrium. New York: Wiley.114p.

DEBREU,G.(1984).Economic Theory in the Mathematical Mode.The American Economic Review, 74(3): 267-278.

DEBREU,G.(1976).”The Application toEconomics ofDifferential Topology And Global Analysis."The Americam Economic Review,66(2): 281287.

DEBREU,G.(1995).Mathematical Economics:Twenty Papers of Gerard Debreu.Cambridge University Press.

DIERKER,E.(1972).”Two remarks on the number of equilibria of an economy”.Econometrica, 40: 951-953.

DIERKER,E.(1990).”Regular economies”.In Eatwell,J \& Milgate,M \&. Newman,P.(eds). The new palgrave : General Equilibrium. New York: W.W. Norton, pp.261-268.

DIEWERT,E.(1974).”Applications of duality theory”. In Intriligator\&Kendrick (eds).Frontiers of Quantitative Economics.Amsterdam:North-Holland.

EATWELL,J.;MILGATE,M.;NEWMAN,P.(1990).The new palgrave : General Equilibrium. New York: W.W. Norton.346p.

EVES, H.(1990). Foundations and Fundamental Concepts of Mathematics. New York: Dover Publications.

FARIAS,T.A.(1987). Estabilidade de equilíbrio competitivo: a relação entre estabilidade dinâmica e expectativa adaptativa. Recife:Dissertação de Mestrado,Universidade Federal de Pernambuco FARIAS,T.A.(1999). Uma abordagem histórica sobre a teoria da estabilidade de equilíbrio competitivo.São Paulo:Tese de Doutorado,Universidade de São Paulo-USP,Estado de São Paulo. 
FARIAS,T.A.(2007). “Teoria da estabilidade de mercados múltiplos."Revista de Economia, 33(2):65-90.

GALE, DAVID. (1960). A Note on Global Instability of Competitive Equilibrium. Office of Naval Research.

GORDON,R.H.(1974). "Negative Quasi-Definiteness and the Global Stability Of General Equilibrium”. Econometrica 42(1): 197-198.

GREEN,J.R.(1974). “The Stability of Edgeworth`s Recontracting Process.” Econometrica, 42(1): 21-34.

HABIBAGAHI,H.;QUIRK,J.(1973). "Hicksiany Stability and Walras`s Law.” Review of Economic. Studies, 49(2): 249-258.

HAHN,F.H.(1961).”A Stable Adjustment Process for a Competitive Economy.” Review of Economic Studies, 29: 62-65.

HAHN , F. H. , (1958) . "Gross Substitutes and the Dynamic Stability of General Equilibrium”. Econometrica, 26: 169-170.

HAHN, F. H. (1962). “On The Stability of Pure Exchange Equilibrium.” International Economic Review, 3: 206-214.

HAHN,F.H;NEGISHI,T. .(1962). "A Theorem on Non - Tâtonnement Stability." Econometrica, 30: 463-469.

HOTAKA,R.(1971). “Some Basic Problems on Excess Demand Functions."Econometrica, 39(2): 305-307.

KAMYIA,K.(1990). “A Globally Stable Price Adjustament Process”. Econometrica, 58: 1481-1486.

LANGE, O. (1944). Price Flexibility and Employment. Bloomington: The Principia Press, 150p.

MCKENZIE,L.W.(1960). "Stability of Equilibrium and the Value of Positive Excess Demand.”Econometrica 28(3): 606-617.

MENEZES,F.M.(1993). "Equilibrium Theory in Infinite Dimensional Spaces.” Revista de Econometria, 13(1): 105-111.

METZLER,L.A.(1945).”The Stability of Multiple Markets:The Hicks Conditions.” Econometrica 13: 277-292.

MILNOR,J.W.(1997).Topology from the differentiable point of view.Revised Edition.New Jersey: Princeton University Press.

MORSE,A.P.(1939).”The behavior of a function on its critical set.”Annals of 
Mathematics 40: 62-70.

MUKHERJI,A.A.(1995). "Locally Stable Adjustment Process.” Econometrica, 63(3): 441-448.

MUKHERJI,A.( 1973). "On the Sensitivity of Stability Results to the Choice of the Numeraire."Review of Economic Studies, 40(3): 427-433.

NEGISHI,T.(1961). “On the Formation of Prices.”International Economic Review 2(1): 123-126.

NEGISHI,T.(1962).”The Stability of a Competitive Economy:A Survey Article." Econometrica 30: 635-669.

NEGISHI,T.(1958). “A Note on the Stability of an Economy where All Goods Are Gross Substitutes.” Econometrica 26: 445-447.

NIKAIDÔ,H.;UZAWA,H.(1960). “Stability Non-Negativity in a Walrasian Tâtonnement Process."International Economic Review, 1(1):50-59.

PALIS Jr,J;MELO,W.(1977). Introdução aos sistemas dinâmicos. Rio de Janeiro: Projeto.Euclides.Impa.

QUIRK,J.P.(1970). “Complementarity and Stability of Equilibrium.” The American Economic Review, 60(3): 358-363.

SAMUELSON,P.A.(1947).The Foundations of Economic Analysis.

Cambrigde:Harvard University Press.380p.

SAMUELSON,P.A.(1941).”The Stability of Equilibrium : Comparative Statics and Dynamics."Econometrica, 9: 97-120,April.

SAMUELSON,P.A.( 1942).”The Stability of Equilibrium:Linear and NonLine Systems."Econometrica,10(1):1-25.

SARD,A.(1942)."The Measure of the critical points of differentiable maps."Bulletin of American Mathematics Society 48: 883-890.

SCARF,H.(1960). "Some Examples of Global Instability of the Competitive equilibrium.” International Economic Review, 1: 157-172.

SMALE, S. (1976a).” A Convergent Process of Price Adjustment and Global Newton Methods." Journal of Mathematical Economics, 3: 107-120.

SMALE,S.(1976b).”Dynamics in General Equilibrium Theory.”

American Economic Association, 66(2): 288-294.

TAAR,DAVID.G.( 1978). "Expectations and Stability with Gross Complements.” Review of Economics Studies, 45(3).

UZAWA,H.( 1962). "On the Stability of Edgeworth's Barter Process.”

International Economic Review, 3: 218-232. 
UZAWA,H.(1961)."The Stability of Dynamic Process."Econometrica, 29(4): 617-631.

WALD,A.(1951). “On some Systems of Equations of Mathematical Economics.” Econometrica 19: 368-403.

WALKER, D. A. (1987). "Walras' Theories of Tâtonnement." Journal of Political Economy, 95(4): 758-774.

WALRAS,L.(1996). Compêndios dos elementos de economia política pura.São Paulo:Nova Cultural.352p.Coleção Os Economistas.

Recebido em: 25 de janeiro de 2008 Primeira resposta em: 20 de junho de 2008 Aceite em: 28 de abril de 2009 